http://jmscr.igmpublication.org/home/ ISSN (e)-2347-176x ISSN (p) 2455-0450 crossref DOI: https://dx.doi.org/10.18535/jmscr/v9i8.03

\title{
Comparison between Gene Expert MTB/RIF Assay and Sputum Microscopy in Diagnosis of Tuberculosis in HIV Patients at Tertiary Care Centre
}

\author{
Authors \\ Dr Lucky Kumar ${ }^{1}$, Dr Kamini Randhawa ${ }^{2}$ \\ ${ }^{1}$ Junior resident, Department of Medicine, IGMC Shimla \\ ${ }^{2}$ Junior resident, Department of Anaesthesia, IGMC Shimla \\ *Corresponding Author \\ Dr Kamini Randhawa \\ Junior resident, Department of Anaesthesia, IGMC Shimla, India
}

\begin{abstract}
Background: HIV is a major contributor to the global burden of disease. The HIV patients are prone to develop many opportunistic infections, consequent to immune-deficiency caused by it. TB is the primary cause of death for 10-15\% of patients with HIV infection. Tuberculosis (TB) is the most common opportunistic infection among people living with HIV. It is also leading cause of death among people living with HIV. People living with HIV are 29 times more likely to develop active TB disease than those without HIV. Patients with HIV pulmonary tuberculosis co-infection are most likely benefitted by Xpert MTB/RIF assay.

Aim and Objective: Comparison between gene expert MTB/RIF assay and sputum microscopy in diagnosis of tuberculosis in HIV patients at tertiary care centre.

Material and Methods: The study was cross sectional study conducted in a period of one year from $1^{\text {st }}$ July 2015 to $30^{\text {th }}$ June 2016 in the department of medicine at Indira Gandhi medical college and hospital. The study population included the HIV positive patient attending ART clinic/Medicine OPD/Pulmonary medicine OPD admitted in medicine ward. Out of these patients with HIV positive, the diagnosis of tuberculosis was established by gene expert or sputum microscopy .Data collected was entered and analysed in excel sheet, using appropriate statistical software and test of significance.

Results: Among 95 patients with HIV infection, the diagnosis of tuberculosis was established in 54 patients after further workup. Seven (26.9\%) out of twenty-six with pulmonary tuberculosis were sputum smear positive on Zeihl-Neelsen staining. All patients with sputumsmear-positive tuberculosis had CD4 count below 200cells/cumm. Nineteen (73.1\%) out of twenty-six with pulmonary tuberculosis were gene xpertpositive. Sixteen (61.5\%) with Xpert MTB/RIF positive tuberculosis had CD4 count below 200cells/cumm. Out of 19 XpertMTB/RIF positive patients only two were rifampicin resistant.

Conclusion: This study has shown that the detection of acid fast bacilli by Xpert MTB/RIF increased by 2.7 times as compared to sputum smear microscopy.
\end{abstract}

\section{Introduction}

HIV is a major contributor to the global burden of disease. In 2010, HIV was the leading cause of disability-adjusted life years worldwide for people aged 30-44 years, and the fifth leading cause for all ages. ${ }^{1}$ According to the Global Tuberculosis 
report 2014 of World Health Organization (WHO), Tuberculosis (TB) remains one of the world's deadliest communicable diseases that is caused by the bacterium Mycobacterium tuberculosis (MTB). ${ }^{2}$ HIV patients are prone to develop many opportunistic infections.

Tuberculosis (TB) is the most common opportunistic infection among people living with HIV. ${ }^{3}$ TB is the primary cause of death for 10 $15 \%$ of patients with HIV infection. ${ }^{4}$ People living with HIV are 29 times more likely to develop active TB disease than those without HIV. Levels of plasma HIV RNA increase in the setting of active TB and decline in the setting of successful TB treatment ${ }^{4}$. In addition, HIV patients have increased the risk for drug-resistant TB.There were an estimated 1.1 million new TB cases among HIV-seropositive patients globally in 2013. At least one-third of the 35 million people living with HIV worldwide are infected with latent TB. TB is also the leading cause of death among people living with HIV, accounting for some 360,000 deaths of HIV-associated TB. As per the Revised National Tuberculosis Control Program (RNTCP), the diagnosis of Pulmonary Tuberculosis is made by sputum microscopy that is fraught with low sensitivity and specificity. ${ }^{5}$ Although smear microscopy is widely used for the rapid diagnosis of $\mathrm{TB}$, it does not detect drug resistance and its sensitivity in individuals coinfected with HIV varies between 40to $60 \%$. $^{6}$ This can result in a large number of cases of active TB disease going undiagnosed. Currently, available techniques are liquid culture systems and molecular line probe assays for rapid detection of MDR-TB, however,their uptake has been limited in many resource-constrained settings, due to their complexity and cost, as well as the need for sophisticated laboratory infrastructure. To overcome these shortcomings as well as limitations of sputum microscopy, WHO recommends the use of Xpert MTB/RIF as a novel diagnostic modality. However, Xpert MTB/RIF is available currently with limited health care facilities in India although it's a cheap, easy, sensitive and specific technology for detection of $\mathrm{TB}$ and resistance to rifampicin associated with it. Patients with HIV-pulmonary tuberculosis coinfection cases are characterized by paucibacillary nature of the disease and AFB smear examination is usually negative. These subset of patients are most likely benefitted by Xpert MTB/RIF examination $^{7}$ a novel diagnostic modality detect M.tuberculosis as well as rifampicin resistance. The development of the Xpert MTB/RIF assay for the GeneXpert platform was completed in 2009 and is considered an important breakthrough in the fight against TB. The Xpert MTB/RIF detects M.tuberculosis as well as rifampicin resistanceconferring mutations using three specific primers and five unique molecular probes to ensure a high degree of specificity. This assay amplifies a $M$. tuberculosis complex-specific region of the rрoB gene, which is probed with molecular beacons to detect the presence of rifampicin resistancedetermining mutations. The assay provides results directly from sputum in less than 2 hours. The present study compared Xpert MTB/RIF and sputum microscopy for the fast detection of tuberculosis and its rifampicin resistance in symptomatic HIV patients with early suspicion of tuberculosis infection at a tertiary care center.

\section{Aims and Objectives}

Comparsion between gene xpert MTB/RIF and sputum microscopy in diagnosis of tuberculosis in HIV patient at tertiary care centre.

\section{Material and Methods}

The study was cross sectional study conducted in a period of one year from $1^{\text {st }}$ July 2015 to $3 o^{\text {th }}$ June 2015 in the department of Medicine at Indira Gandhi Medical College and Hospital, Shimla. The study population included the HIV positive patients attending the ART clinic/Medicine OPD/Pulmonary Medicine OPD/admitted in Medicine ward from $1^{\text {st }}$ July 2015 to $30^{\text {th }}$ June 25, 2015. All HIV positive patients admitted in Medicine ward and attending ART clinic/ Medicine OPD/Pulmonary Medicine OPD with 
any of following complaints i.e. fever, cough, night sweat, weight loss, shortness of breath and age more than 18 yrs were included in study. Those who didn't fulfill inclusion criteria and who didn't give consent were excluded from study. Ethical Considerations to be consider and whole information regarding study kept confidential and anonymity maintained. Informed written consent to be taken from all patients included in the study. The consent for HIV testing taken before collecting the sample from the patients as per National Program. For diagnosis of HIV five milliliter of venous blood was collected by venupuncture and transferred aseptically from syringe into sterile test tube. The blood was allowed to clot for 3 minutes at room temperature and then clot was gently broken by using a sterile Pasteur pipette .Test tube was then centrifuged at $1200 \mathrm{G}$ for 10 minutes to separate serum. Diagnosis of HIV was made as per national for HIV testing based on E.R.S. (ELISA rapid and simple) using commercially available kits .CD4 cells count was done by fluorescent activated cell counter after the selection of patients according to inclusion criteria, relevant history was taken, physical and systemic examination was done. Then the patients were subjected to investigations including RBS, hemogram, CD4 count, renal functions tests, liver functions tests, chest $\mathrm{X}$-rays and sputum smear microscopy at Direct Observed Treatment (DOT) Center, Xpert MTB/RIF. Extracted or induced sputum samples in sterile container with screw cap were accepted was subjected to Xpert MTB / RIF.

1) To minimum sample of $1 \mathrm{ml}$ was added twice quantity of buffer.

2) Shaked vigorously and stand for 15 minutes.

3) $2 \mathrm{ml}$ of homogenized sample was added to the cartridge.

4) Result was obtained in 2 hour time.

Diagnosis of tuberculosis was established by

1) Isolating acid fast bacilli (AFB) from given specimen by Z.N. staining or Gene Xpert or culture
2) Histopathological demonstration of typical caseous granulomatous reaction.

3) Clinical profile and response to ATT.

4) Radiological features suggestive of tubercular lesions.

5) Pleural /asitic fluid analysis showing evidence of lymphocytic exudative effusion and CSF showing lymphocytic with hypoglycorrhachia (low CSF glucose).

Statistical analysis: Data collected was entered and analyzed in excel sheet, using appropriate statistical software and test of significance.

\section{Results}

This was a cross sectional study conducted in the department of Medicine, IGMC Shimla from June 2015 to May 2016. We evaluated 95 HIV patients with symptoms suggestive of tuberculosis (e.g. cough, fever,weightloss,nightsweat, shortness of breath) from the department of Medicine, Pulmonary medicine,ART center. Out of these 95 patients with HIV infection, the diagnosis of tuberculosis was established in 54 patients after further workup.

\section{Age and Sex Distribution}

The mean age of patients was 40.4 yrs (median 39 yr, range 21-60 years). The majority $(53.7 \%)$ of patients were in the age group of 31-40 years. Three (5\%) were the age group 18-30 years and $16(29.6 \%)$ patients in the group of $41-50$ years. Six patients $(11.1 \%)$ were above 50 years. Out of 54 patients, 40 were males and 14were females. Table -1 shows the distribution of the patients.

Table 1 Age Distribution Total No. Patients 54

\begin{tabular}{|l|c|c|}
\hline $\begin{array}{l}\text { Age Group } \\
\text { In Years }\end{array}$ & $\begin{array}{c}\text { No. Of } \\
\text { Patients }\end{array}$ & Percentage\% \\
\hline $18-30$ & 3 & 5 \\
\hline $31-40$ & 29 & 53.7 \\
\hline $41-50$ & 16 & 29.6 \\
\hline$>50$ & 6 & 11.1 \\
\hline
\end{tabular}

\section{Distribution of Sputum for AFB Positive and} AFB Negative Pulmonary Tuberculosis

Seven $(26.9 \%)$ out of twenty-six with pulmonary tuberculosis were sputum smear positive on Zeihl- 
Neelsen staining. All patients with sputumsmearpositive tuberculosis had CD4 count below 200cells/cumm. Table 2 shows the distribution of sputum positivity in patients with pulmonary tuberculosis.

\section{Table 2}

Total Patients 26

Distribution of Sputum for AFB Positive/

Negative PTB

\begin{tabular}{|l|c|c|}
\hline CD 4 & $\begin{array}{c}\text { SPUTUM(+) } \\
\text { PTB (7) }\end{array}$ & $\begin{array}{c}\text { SPUTUM (-) } \\
\text { PTB (19) }\end{array}$ \\
\hline$<50$ & 3 & 5 \\
\hline $51-100$ & 3 & 2 \\
\hline $101-150$ & 1 & 4 \\
\hline $150-200$ & 0 & 3 \\
\hline$>201-250$ & 0 & 3 \\
\hline $251-500$ & 0 & 1 \\
\hline$>500$ & 0 & 0 \\
\hline
\end{tabular}

\section{Distribution of Sputum for Gene Xpert Positive Pulmonary Tuberculosis:}

Nineteen (73.1\%) out of twenty-six with pulmonary tuberculosis were gene xpert positive. Sixteen (61.5\%) with Xpert MTB/RIF positive tuberculosis had CD4 count below 200cells/cumm. Seven patients out of total twenty-six patients, which were negative for both smear for AFB and gene xpert as well, were diagnosed on the basis of clinical symptomatology, chest X-ray findings, raised ESR and response to treatment. All Xpert MTB/RIF positive cases except one were positive on Mycobacteria Growth Indicator Tube (MGIT) and Lowenstein-Jensen (LJ) medium. Out of 19 Xpert MTB/RIF positive patients only two were rifampicin resistant. Thus the detection of acid fast bacilli by Xpert MTB/RIF increased by 2.7 times as compared to sputum smear microscopy. Table 3 shows the distribution of sputum positivity for Xpert MTB/RIF in the patients of pulmonary tuberculosis
Table 3 Distribution of Sputum For Xpert MTB/RIF Positive

Total No. Patients 19

\begin{tabular}{|l|c|c|}
\hline CD4 COUNT & $\begin{array}{c}\text { GENE XPERT (+) } \\
(19)\end{array}$ & $\begin{array}{c}\text { GENE XPERT (-) } \\
(9)\end{array}$ \\
\hline$<50$ & 5 & 3 \\
\hline $51-100$ & 4 & 2 \\
\hline $101-150$ & 4 & 1 \\
\hline $151-200$ & 3 & 2 \\
\hline $201-250$ & 2 & 1 \\
\hline $251-500$ & 1 & 0 \\
\hline$>500$ & 0 & 0 \\
\hline
\end{tabular}

\section{Discussion}

Tuberculosis remains a major public health problem, particularly in low and middle-income countries. Over $95 \%$ of TB deaths occur in low and middle-income countries. Six countries account for $60 \%$ of the total, with India leading the count, followed by Indonesia, China, Nigeria, Pakistan and South Africa. TB is a leading killer of HIV-positive people in $2015^{8}$. The spread of multidrug-resistant TB (MDR-TB) and its detrimental convergence with HIV infection and the unavailability of rapid diagnostic tools have contributed to the failure of global TB control $^{9-11}$. Moreover, through altering the clinical and bacteriological presentations, HIV infection has contributed to the delay in diagnosing active TB disease, which leads to the increased morbidity, mortality and enhancement of the rate of transmission within communities. ${ }^{12-14}$ The diagnosis of tuberculosis has never been easy as there is a wide range of clinical features. Heightened clinical awareness remains the mainstay of diagnosis. Delay in TB diagnosis, due to limited screening procedures, inaccuracy of diagnostic algorithms and lack of adequate laboratory facilities, is recognized as a major limitation for TB control efforts in correctional settings. ${ }^{13}$ Xpert MTB/RIF, a new nucleic acid based amplification technology, offers rapid and accurate diagnostic results from biological specimens with minimal staff training requirements. ${ }^{15-17} \mathrm{~A}$ recent review of published evaluation reports confirmed that the use of Xpert $\mathrm{MTB} / \mathrm{RIF}$ as an initial test replacing smear 
microscopy was highly accurate. ${ }^{16}$ In 2010 and with the encouraging initial evaluation reports, the WHO endorsed Xpert MTB/RIF to be used as the initial TB diagnostic test for individuals with HIV-infection or those suspected of having MDRTB. ${ }^{18}$ This study was designed to evaluate tuberculosis in HIV patients usingXpert MTB/RIF assay for early and fast detection of tuberculosis in HIV positive patients and also to look for rifampicin resistance, along with various other techniques in atertiary care setting. The symptomatic patients were evaluated for tuberculosis at the onset of study or subsequently during follow-up visits with the use of smear microscopy, Xpert MTB/RIF for sputum, pleural fluid, CSF, ascitic fluid, Lymph node aspirate, ultrasonography abdomen and CT scan, if needed. Tuberculosis is the most common opportunistic infection in HIV-positive patients. Globally, about one-third of HIV-positive patients are co-infected with TB. About 5\% of new TB cases in India occur in people HIV with infection. ${ }^{4}$ In endemic countries, TB infection is usually acquired in childhood. Because of robust immunity, it remains as latent TB infection (LTBI). The lifetime risk of reactivation of latent TB in HIV-negative persons is $10 \%$ but in HIV-positive persons, the risk is $5 \%$ to $10 \%$ yearly, leading to a lifetime risk of $50 \%{ }^{19}$ In a study by Agarwal et al, $29.1 \%$ of HIVinfected patients accessing highly active antiretroviral therapy (HAART) had concurrent active TB and treatment of both conditions together lead to an improved outcome.$^{20}$

HIV decreases macrophages and CD4 cells, which are the main cells that provide immunity. In HIVpositive persons, there is an increased risk of primary $\mathrm{TB}$, rapid progression of $\mathrm{TB}$ and reactivation of TB. There is also an increased incidence of extrapulmonary TB (EPTB), disseminated TB and immune reconstitution TB in patients on HAART. In our study, a total of 95 patients were evaluated, and HIV-TB co-infection was diagnosed in $54(56.84 \%)$ patients. Bhagywati Devi etal. ${ }^{21}$ also reported HIV-TBcoinfection in $55 \%$ of their patients. Ghiya et al. ${ }^{22}$ similarly reported HIV-TB co-infection in $49.2 \%$ of HIV cases in their study. Sharma et al. ${ }^{23}$ reported TB-HIV co-infection in $33.2 \%$ of HIV patients. Our study included only symptomatic patients and most of them were referred cases due to lack of rapid and sensitive diagnostic facilities at peripheral hospitals. This may explain the higher incidence of TB in these HIV patients coming to our tertiary care center. The majority of patients $(68.9 \%)$ in our study were in the age group of 31 to 40 years. Kamath etal. ${ }^{24}$ also reported similar age group of 31 to 45 years in $61.3 \%$ of patients in their study. The mean age of HIV-TB co-infection was 36 yrs in the study by Sharma et al. $^{23}$ The majority of patients i.e. $70.07 \%$ were males. About $75.3 \%$ and $81.3 \%$ of HIV-infected patients were male in the study by Kamath etal. ${ }^{24}$ and Sharma et al. ${ }^{23}$ respectively. Males were more commonly affected than females due to their occupational (like drivers) requirements and need to travel to distant places in the country for their business.

Out of 26 pulmonary tuberculosis patients, seven (26.92\%) were diagnosed by smear microscopy, nineteen patients $(73.07 \%)$ were diagnosed by Xpert MTB/RIF assay of sputum sample, seven patients were diagnosed on the basis of radiological features and response to antitubercular treatment. All 19 patients except one which was diagnosed by Xpert MTB/RIF, were confirmed by MGIT and LJ culture. Out of 19 XpertMTB/RIF positive patients only two were rifampicin resistant. The Xpert MTB/RIF assay has increased the case detection rate in pulmonary tuberculosis by $46.15 \%$ as compared to sputum smear microscopy.

\section{Summary and Conclusion}

The study revealed following features. The most common affected age group was 31-40 years. Males were affected more than females. The most common affected professional group was of drivers. Out of 95 patients for symptoms, tuberculosis was diagnosed in $54(56.84 \%)$ patients. Out of the fifty-four patients with HIV- 
TB coinfection 27 patients (49.99\%) had CD4cellcount $<100 /$ cumm and 38 patients (70.36\%) had CD4cellcount $<200 /$ cumm. This shows that tubercular co-infection is more common in HIV-infected with advancing immunosuppression. Out of 26 pulmonary tuberculosis patients, seven $(26.92 \%)$ were diagnosed by smear microscopy, nineteen patients (73.07\%) were diagnosed by xpert MTB/RIF for sputum. So xpert MTB/RIF increased detection of sputum positive tuberculosis by $46.15 \%$. Out of $19 \mathrm{XpertMTB/RIF}$ positive patients only two were rifampicin resistant. The present study in tertiary care center setting is in favor of WHO endorsement that Xpert MTB/RIF to be used as the initial TB diagnostic test for individuals with HIV-infection. Thus Xpert MTB/RIF has a definite role in early detection of pulmonary tuberculosis among patients suffering from HIV disease. It not only establishes early diagnosis of tuberculosis, but also provides information regarding rifampicin resistance as well

\section{References}

1. Ortblad KF, Lozano R, and Murray CJ.The burden of HIV: insights from the GBD2010. AIDS. 2013; 27: 2003-2017.

2. World Health Organization. Global tuberculosis report 2014. Geneva: WHO 2014.Availablefrom:URL:http://apps.who. int/iris/bitstream/10665/137094/1/9789241 564809_eng.pdf?ua=1. (Last accessed on 18.16.2016).

3. Londhey VA.HIV and Tuberculosis-A "Cursed Duo" in theHAART Era, J Assoc Physicians India 2009; 57: 681-82.

4. Longo Dan L, Kasper Dennis L, Jameson J et al. Harrison's Principles of Internal Medicine, $19^{\text {th }}$ edMcGraw-Hill, 2015: 1216-54.

5. Arzu N. Zeka, Sezai Tasbakan, Cengiz Cavusoglu. Evaluation of the Gene Xpert MTB/RIF Assay for Rapid Diagnosis of Tuberculosis and Detection of Rifampin Resistance in Pulmonary and
Extrapulmonary Specimen Journal of Clinical Microbiology. Dec, 2011; 49 (12) 4138-41.

6. Longo Dan L, Kasper Dennis L,Jameson J et al. Harrison's Principles of Internal Medicine .19th edMcGraw-Hill2015:1133

7. World Health Organization: Automated real-time nucleic acid amplification technology for rapid and simultaneous detection of tuberculosis and rifampicin resistance: Xpertmtb/rif system. Policy statement 2011.Available from URL http://whqlibdoc.who.

int/publications/2011/9789241501545_eng .pdf.

8. Southwick F. Chapter 4 Pulmonary Infections. Infectious Diseases: A clinical Short Course. $2^{\text {nd }}$ ed McGraw-Hill Medical Publishing Division,2007; 313-14.

9. Lawn SD, Zumla AI. Tuberculosis. Lancet. $2011 \mathrm{Jul}$ 2;378(9785):57-72.

10. Zumla A, Raviglione M, Hafner R et al. Tuberculosis. N Engl J Med. 2013 Feb 21;368(8):745-55.

11. Corbett EL, Marston B, Churchyard GJ et al. Tuberculosis in sub-Saharan Africa: opportunities, challenges, and change in the era of antiretroviral treatment. Lancet 2006; 367: 926-37.

12. Golub JE, Mohan CI, Comstock GW et al. Active case finding of tuberculosis: historical perspective and future prospects. Int J Tuberc Lung Dis. 2005 Nov;9(11): 1183-203.

13. Wood R,Middelkoop K, Myer L et al. Undiagnosed tuberculosis in a community with high HIV prevalence: implications for tuberculosis control. Am J RespirCrit Care Med. 2007 Jan 1;175(1):87-93.

14. Davis JL, Worodria W, Kisembo $\mathrm{H}$ et al. Clinical and radiographic factors do not accurately diagnose smear-negative tuberculosis in HIV-infected inpatients in Uganda: a cross-sectional study. PLoS One. 2010 Mar 26;5(3):e9859. 
15. Boehme CC, Nabeta P, Hillemann D et al. Rapid Molecular Detection of Tuberculosis and Rifampin Resistance. N Engl J Med. 2010;363:1005-15.

16. Steingart K, Sohn H, Schiller I et al. Xpert ${ }^{\circledR}$ MTB / RIF assay for pulmonary tuberculosis and rifampicin resistance in adults. Cochrane Database Syst Rev. 2013 Jan 31;(1):CD009593.

17. Chang K, Lu W, Wang J et al. Rapid and effective diagnosis of tuberculosis and rifampicin resistance with Xpert MTB/RIF assay: a meta-analysis. J Infect. 2012 Jun;64(6):580-8.

18. World Health Organization (WHO) (2011) Rapid Implementation of the Xpert MTB / RIF diagnostic test: technical and operational "How-to"; practical considerations. Geneva.

19. Guidelines for prevention and management of common opportunistic infections/malignanciesamong HIV infected adults and adolescents. NACO, 2007; 12-17.

20. Agarwal U, KumarA, Behera D. Profile of HIV-Associated Tuberculosis at a Tertiary Institute in Setting of Free Anti-Retroviral Therapy, J AssocPhysicians India 2009;57: 685-690

21. Bhagyabati S, Naorem S, Singh J et al. HIV and TB Co-infection. Journal, Indian Academy of Clinical Medicine 2005;6(3):220-223.

22. Ghiya R, Naik E, Casanas B et al. Clinicalepidemiological profile of HIV/TB coinfected patients in Vadodara, Gujarat. Indian journal of sexually transmitted diseases 2009; 30:10-15.

23. Sharma SK, Kohli M, Yadav RN et al. Evaluating the Diagnostic Accuracy of Xpert MTB/RIF Assay in Pulmonary Tuberculosis. PLoS ONE 2015;10(10): e0141011.
24. Kamath R, Sharma V, Pattanshetty S et al. HIV-TB coinfection: Clinicoepidemiological determinants at an antiretroviral therapy center in Southern India. Lung India 2013; 30(4):302-06. 